acuity which appears in the text of the paperthat is, " $6 / 18$ or worse in the better eye, both eyes using best correction."

Our error was to attribute this definition to the WHO both in the text and in the heading of table I. We apologise for any confusion we may have caused to readers.

David H Stone

Greater Glasgow Health Board, Glasgow

Pfizer (Central Research),

Sandwich, Kent ${ }^{1}$ World Health Organisation, The Prevention of Blindness,
WHO Technical Report Series No 518. Geneva,
WHO, 1973.

\section{Treatment of rheumatoid arthritis}

SIR,-I read with intersst the letter from Dr R Luukkainen and others (25 November, p 1501).

I quite agree that gold or other diseasemodifying drugs need to be used in several situations in the treatment of rheumatoid arthritis. Indeed, in the article (14 October, p 1068) I listed four different indications. The first covers the situation described in their letter: "The most common group includes those patients with widespread active disease, usually in the first year of disease, whose disease activity cannot be controlled despite the trial of several non-steroidal antiinflammatory drugs in effective dosage." The second group was those with progressive radiological damage, the third group those with systemic features, and the fourth group those with long-standing disease but with continuing activity. This will show that we are in complete agreement.

I am not sure that it is correct to say that the result is worse if gold treatment is initiated later. One can be categorical and say that patients started on gold later in the course of the disease require a greater total dose (average $920 \mathrm{mg}$, range $325-2780 \mathrm{mg}$ ) given as $50-\mathrm{mg}$ injections at weekly intervals before improving in comparison with those started earlier (average $591 \mathrm{mg}$, range $160-1180 \mathrm{mg}$ ). The quality of the response may be comparable.

Northwick Park Hospital and Clinical Research Centre, Harrow, Middx

'Gumpel, J M, Rheumatology and Rehabilitation, 1976,
15, 217.

\section{Metoclopramide in young children}

SIR,-Despite the recent attention drawn to the adverse toxic effects of metoclopramide in children the drug continues in common usage by family practitioners in this region.

Drs J A Sills and E J Glass (5 August, p 431) mention the narrow margin between toxic and therapeutic dosage of metoclopramide and have reported two children who required admission to hospital following "convulsions." Recently we have seen a further two children whose initial symptoms of pyrexia and vomiting were treated with metoclopramide and antibiotics. Within six hours of metoclopramide administration both febrile children were admitted to hospital with severe extrapyramidal dystonia, including opisthotonos and marked neck retraction. As the possibility of meningitis could not be excluded both children had lumbar punctures that yielded a normal cerebrospinal fluid. While the definitive diagnosis of dystonic reaction could have been made initially, the outcome of missing a purulent meningitis is too serious to omit a spinal tap. Conversely, although metoclopramide produces a satisfying symptomatic relief of vomiting in many instances, as Dr A Bloch (14 October, p 1092) points out, the resultant effect has actually masked the diagnosis of meningitis in three infants.

The known indications for the use of this efficacious drug have been well documented ${ }^{1}$ and the danger of overdosage outlined. If the daily dose of metoclopramide exceeds $0.5 \mathrm{mg} /$ $\mathrm{kg}$ /day the incidence of dystonic reactions is markedly increased. There is the all too common temptation by parents to administer more than the prescribed dose in order to abate the symptoms more rapidly. Simpler and safer measures are recognised that are equally efficacious in preventing vomiting in children. The use of metoclopramide for infants and young children should be discouraged and more attention paid to the primary diagnosis rather than to the symptoms it produces.

G J REYNOLDS

University Department of Child Health,

Alder Hey Children's Hospital,

A.iverpool

${ }^{1}$ Nimmo, W S, Prescribers fournal, 1977, 17, 90.

\section{Management of defects of vision in} early childhood

SIR,-In your series "ABC of Ophthalmology" Mr P A Gardiner states (18 November, p 1411) that "judging distances . . . requires much experience and experiment." While I would not dispute that the ability to make absolute judgments of distance develops during childhood, there is evidence to suggest that even very young infants may be able to judge relative distance. Some years ago Bower demonstrated experimentally ${ }^{1}$ that infants less than 2 months of age were able to discriminate successfully between different distances when the only cue available to them was that of motion parallax, the term used to refer to the fact that the images of objects at different distances move across the retina at different speeds during lateral movement of the head.

We can detect whether an object is in front of or behind the plane of fixation by relying on stereoscopic vision. The mechanism underlying stereopsis can be illustrated by means of what are known as random dot stereograms. A pair of stereograms is constructed such that dots generated randomly by computer appear more or less identically in each member of the pair. The only difference between the two stereograms is that in one a central region of dots is shifted slightly in a horizontal direction relative to its position in the other. Viewed monocularly each stereogram appears as a random array of dots. If, however, each member of the pair is viewed by a different eye by means of a stereoscope of cross-polarised filters the central region of displaced dots appears to stand out dramatically in depth. ${ }^{2}$ This demonstrates that stereopsis depends upon the horizontal disparity between the two images of the same object at the left and right eyes. Recently two out of four 2-month-old infants gave evidence of being able to discriminate between sets of random dot stereo-pairs having different horizontal disparities. ${ }^{3}$ As these infants showed no differential response to sets of stereograms differin in vertical disparity it is difficult to resist the conclusion that some degree of stereoscopic vision may be present even at this early age. In support of this argument it may be noted that, like normal adults and children but unlike stereo-defective subjects, babies aged as little as 2 months show enhanced visual evoked potentials with binocular as compared to monocular viewing. ${ }^{4}$ At the very least this implies that information from the two eyes is integrated at some level in the immature brain.

Neurophysiological research with cats and monkeys has shown that the vast majority of neurones in the striate cortex can be stimulated by input presented to either the left or the right eye. In the adult cat or monkey such binocular cortical neurones fire maximally when separate stimuli fall simultaneously on the two retinas. However, the exact position of the stimuli is crucial and differs slightly for one eye relative to the other, the preferred disparity differing for different cortical cells. These binocular cells are therefore thought to act as disparity detectors and are thus clearly implicated in the phenomenon of stereopsis. ${ }^{5}$

The link between such work and humans is this. Binocular cortical neurones are present as soon as the animal opens its eyes. Suturing of one eye from birth or a surgically induced squint, however, results in a drastic reduction in the proportion of binocularly driven neurones encountered during later microelectrode exploration. ${ }^{6}$ As is well known, human strabismic patients rarely possess stereoscopic vision even if the defect was surgically corrected at an early age. Behavioural testing of patients having strabismic or anisometropic amblyopia reveals that, unlike most normal subjects, they show little if any transfer of certain monocularly generated perceptual effects from the viewing to the non-viewing eye. This is consistent with the notion that such patients are deficient in cortical binocular neurones as a result of their early anomalous visual experience.?

It appears that the developing brain can be modified by visual experience other than through amblyopic abnormality. Shlaer raised kittens with a prism permanently in front of one eye such that the scene viewed by that eye was shifted relative to the other eye. ${ }^{8}$ Subsequent recording with microelectrodes suggested that binocular cortical neurones had adjusted to the disparity induced by the prism. More recent experiments have confirmed this early plasticity of the visual system. Given, then, that cortical binocularity drops out in cases of squint yet is modified so as to accommodate relatively less severe prism-induced distortions, it seems likely, in view of the evidence discussed above, that the human infant is born with a functional binocular visual system that is able to operate only within certain predetermined limits. Gradually the system becomes more finely tuned in accordance with the infant's experience. This would, of course, be adaptive in allowing the brain to compensate for changes in ocular alignment and inter-eye distance as the infant grows older.

\section{Psychology Department}

University College,

Swansea

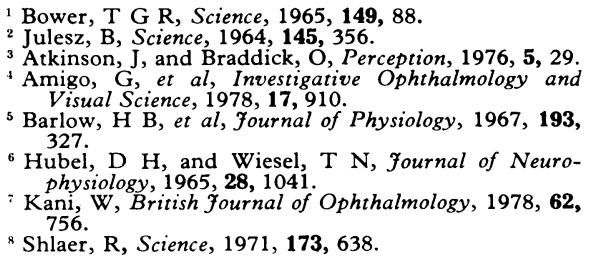

Alan A Beaton

\section{Postanaesthetic oxygen}

SIR,-Your recent leading article on this subject (25 November, p 1452) suggests that oxygen "should be prescribed in a known concentration for a specific time for those who need it." The elderly, patients with additional 